\title{
Mature brain-derived neurotrophic factor and its receptor TrkB are upregulated in human glioma tissues
}

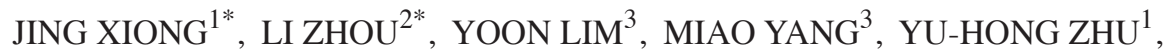 \\ ZHI-WEI LI ${ }^{1}$, DENG-LI FU ${ }^{1}$ and XIN-FU ZHOU ${ }^{3,4}$

\begin{abstract}
${ }^{1}$ Department of Neurology, Second Affiliated Hospital of Kunming Medical University, Kunming, Yunnan 650101;
${ }^{2}$ The First People's Hospital of Yunnan, Kunming, Yunnan 650000, P.R. China; ${ }^{3}$ School of Pharmacy and Medical Sciences, University of South Australia, Adelaide, SA 5000, Australia; ${ }^{4}$ Key Laboratory of Stem Cells and Regenerative Medicine,

Institute of Molecular and Clinical Medicine, Kunming Medical University, Kunming, Yunnan 650101, P.R. China
\end{abstract}

Received August 13, 2014; Accepted April 17, 2015

DOI: $10.3892 / \mathrm{ol} .2015 .3181$

\begin{abstract}
There are two forms of brain-derived neurotrophic factor (BDNF), precursor of BDNF (proBDNF) and mature BDNF, which each exert opposing effects through two different transmembrane receptor signaling systems, consisting of p75 neurotrophin receptor (p75NTR) and tyrosine receptor kinase B (TrkB). Previous studies have demonstrated that proBDNF promotes cell death and inhibits the growth and migration of $\mathrm{C} 6$ glioma cells through $\mathrm{p} 75 \mathrm{NTR}$ in vitro, while mature BDNF has opposite effects on C6 glioma cells. It is hypothesized that mature BDNF is essential in the development of malignancy in gliomas. However, histological data obtained in previous studies were unable distinguish mature BDNF from proBDNF due to the lack of specific antibodies. The present study investigated the expression of mature BDNF using a specific sheep monoclonal anti-mature BDNF antibody in 42 human glioma tissues of different grades and 10 control tissues. The correlation between mature BDNF and TrkB was analyzed. Mature BDNF expression was significantly increased in high-grade gliomas, and was positively correlated with the malignancy of the tumor and TrkB receptor expression. The present data have demonstrated that increased levels of mature BDNF contribute markedly to the development of malignancy of human gliomas through the primary BDNF receptor TrkB.
\end{abstract}

Correspondence to: Dr Jing Xiong, Department of Neurology, Second Affiliated Hospital of Kunming Medical University, 374 Dianmian Road, Kunming, Yunnan 650101, P.R. China

E-mail: xiongjing3210@126.com

Professor Xin-Fu Zhou, School of Pharmacy and Medical Sciences, University of South Australia, Frome Road, Adelaide, SA 5000, Australia

E-mail: xin-fu.zhou@unisa.edu.au

${ }^{*}$ Contributed equally

Key words: brain-derived neurotrophic factor, glioma, human, tyrosine receptor kinase $\mathrm{B}$

\section{Introduction}

Brain-derived neurotrophic factor (BDNF), a member of the neurotrophin family, is regarded as an oncogenic factor in tumorigenesis, proliferation and survival, and is associated with a poor prognosis in nervous system tumors (1-3). Malignant glioma cells are extremely infiltrative, and patients with malignant glioma demonstrate a short survival time and poor prognosis. Neurotrophin genes are widely expressed in 24 cell lines derived from human malignant gliomas, and the BDNF gene is the most abundantly expressed (4). There are two forms of BDNF, consisting of precursor of BDNF (proBDNF) and mature BDNF, in the central nervous system (CNS) (5). ProBDNF is synthesized and subsequently cleaved, either intracellularly, by prohormone convertases and furin, or extracellularly, by plasmin and matrix metalloproteinases (MMPs), to generate mature BDNF $(6,7)$. Mature BDNF and proBDNF exert opposing effects through two different transmembrane receptor signaling systems, consisting of tyrosine receptor kinase B (TrkB) and p75 neurotrophin receptor (p75NTR) to modulate cell apoptosis (8-10), long-term depression (11) and synaptic plasticity (12-15).

Specific antibodies have previously been generated against mature BDNF and proBDNF (5). In previous studies $(16,17)$, the proBDNF/p75NTR pathway has been demonstrated to promote cell death and inhibit growth and migration in C6 glioma cells through p75NTR in vitro, while mature BDNF demonstrates the opposite effect on C6 glioma cells. It is hypothesized that mature BDNF plays an essential role in the development of gliomas towards malignancy. However, histological data in previous studies were unable distinguish mature BDNF from proBDNF due to the lack of specific antibodies (1-3). In the present study, the expression of mature BDNF was examined in human glioma tissue of various grades using a specific antibody against mature BDNF.

\section{Materials and methods}

Patients. All 42 patients in the present study were enrolled from the Department of Neurosurgery and the Department of Oncology at the Second Affiliated Hospital of Kunming Medical 
University (Kunming, Yunnan, China). The use of human tissue in the present study was approved by the Ethics Committee of Kunming Medical University (Kunming, Yunnan, China), and written informed consent was obtained from all patients. All tumor specimens were classified and graded by two independent pathologists, with full diagnostic agreement, according to the 2007 World Health Organization (WHO) classification of tumors of the CNS (18). The glioma samples were classified as follows: 3 samples, all pilocytic astrocytomas, were classified as grade I; 18 samples, all diffuse astrocytomas, were classified as grade II; 13 samples, all anaplastic astrocytomas, were classified as grade III; and 8 samples, all glioblastoma, were classified as grade IV. For convenience, all gliomas were divided into two groups for analysis. The low-grade glioma group consisted of tissues from 8 male and 13 female patients with grade I and II glioma (mean age, $35.57 \pm 14.95$ years), and the high-grade group consisted of tissues from 13 male and 8 female patients with grade III and IV glioma (mean age, $47 \pm 15.42$ years). In total, 19 samples were resected from the frontal lobe, 12 from the temporal lobe, 5 from the parietal lobe, 3 from the occipital lobe and 3 from the ventricles. There were 9 tumors that involved multiple lobes.

In addition, 10 non-neoplastic brain tissues obtained from 8 males and 2 females (mean age, $41.30 \pm 14.49$ years) were used as control tissues. In total, 3 patients underwent lobe resection for epilepsy, 4 for brain trauma, 2 for hypertensive cerebral hemorrhage and 1 for internal decompression, the sample from which consisted of normal tissue located near a tumor. In addition, 4 control tissues were acquired from the frontal lobe ( 3 patients with brain trauma, 1 patient with glioma who underwent internal decompression), 4 from the temporal lobe (3 patients with epilepsy who underwent lobe resection, 1 patient with brain trauma who underwent internal decompression), 1 from the parietal lobe (1 patient who underwent internal decompression for hypertensive cerebral hemorrhage) and 1 from the cerebellum (1 patient who underwent internal decompression for hypertensive cerebral hemorrhage).

Immunohistochemistry. Small fragments of tissue were fixed in $10 \%$ neutral buffered formalin and embedded in paraffin for immunohistochemistry (IHC) of mature BDNF and TrkB. Single-labeling IHC was performed as described in a previous study (16). Sheep monoclonal anti-mature BDNF (2 $\mu \mathrm{g} / \mathrm{ml}$; laboratory of Professor Xin-Fu Zhou, School of Pharmacy and Medical Sciences, University of South Australia, Adelaide, Australia) and rabbit anti-TrkB (dilution, 1:1000; Catalog no. 07-225; EMD Millipore, Billerica, MA, USA) primary antibodies were used as previously described (17). IHC staining was assessed semi-quantitatively by measuring the intensity of the staining and the number of positive cells, as previously described (16).

Reverse transcription-quantitative polymerase chain reaction (RT-qPCR). The expression levels of the BDNF and TrkB genes in the control brain tissues and glioma tissues of various malignancy grades were determined by RT-qPCR. Quantitative data were normalized relative to $A C T B$. The $5^{\prime}-3^{\prime}$ primer sequences were as follows: $B D N F$ forward, TACTTTGGTTGCATGAAGGCTGCC and reverse, ACTTGACTACTGAGC ATCACCCTG; TrkB forward, AGGGCAACCCGCCCA
CGGAA and reverse, GGATCGGTCTGGGGAAAAG; and $A C T B$ forward, CGGGAAATCGTGCGTGAC and reverse, TGGAAGGTGGACAGCGAGG. All primers were synthesized by Invitrogen (Carlsbad, CA, USA). The total RNA extraction from tissue samples and reverse transcription were performed as described (16). The data were analyzed using the $2^{-\Delta \Delta C t}$ method (19).

ELISA for mature BDNF and TrkB. The tissue samples were homogenized, and levels of mature BDNF in the homogenate were determined by a highly specific, mature BDNF ELISA kit, as previously described (20). The procedure is highly specific for mature BDNF only and does not detect proBDNF or the other neurotrophins, such as neurotrophins- 3 and -4 and nerve growth factor. Levels of TrkB in the protein samples were determined using a commercial human TrkB kit (Sino Biological, Inc., Beijing, China), according to the manufacturer's instructions.

Statistical analysis. The Kruskal-Wallis test, Mann-Whitney $\mathrm{U}$ test and Spearman's rank correlation coefficient were used to compare the differences between the control brain samples and glioma samples. $\mathrm{P}<0.05$ was considered to indicate a statistically significant difference.

\section{Results}

Expression of mature BDNF in human glioma tissues. Immunostaining for mature BDNF was detected in the neuronal cytoplasm of control tissues (Fig. 1A) and cytoplasm of glioma cells (Fig. 1B and C). Strong staining for mature BDNF occurred in the cytoplasm of the high-grade glioma tissues (Fig. 1C). Compared with low-grade glioma and control tissues, the semi-quantitative analysis revealed that mature BDNF immunostaining in high-grade glioma was increased (Fig. 1D; P<0.001). The RT-qPCR (Fig. 1E) analysis also revealed the increased $B D N F$ mRNA levels in high-grade glioma tissues $(\mathrm{P}=0.003)$. These results were further supported by data obtained from ELISA (Fig. 1F). The expression of mature BDNF in low-grade gliomas was significantly increased 1.98-fold compared with the normal control tissue specimens $(\mathrm{P}<0.001)$. Notably, the expression of mature BDNF in high-grade gliomas was significantly increased 4.14-fold compared with low-grade gliomas $(\mathrm{P}<0.001)$.

Expression of TrkB in human glioma tissues. TrkB immunostaining was evidently present in the cytoplasm of neurons in the control tissues (Fig. 2A). There was a weak immunostaining for TrkB in the cytoplasm of the tumor cells of low-grade gliomas (Fig. 2B). By contrast, strong TrkB immunostaining was observed in the cytoplasm of the high-grade glioma cells (Fig. 2C). The semi-quantitative analysis revealed that TrkB immunostaining in high-grade gliomas was significantly increased compared with low-grade glioma and control tissues (Fig. 2D; P<0.001). The RT-qPCR analysis revealed that the expression of TrkB mRNA was increased in high-grade glioma tissues, which was consistent with the results of immunostaining (Fig. 2E; $\mathrm{P}=0.032$ ). An ELISA assay for TrkB revealed that the concentration of TrkB was significantly increased in high-grade gliomas (Fig. 2F; $\mathrm{P}<0.001$ ). 

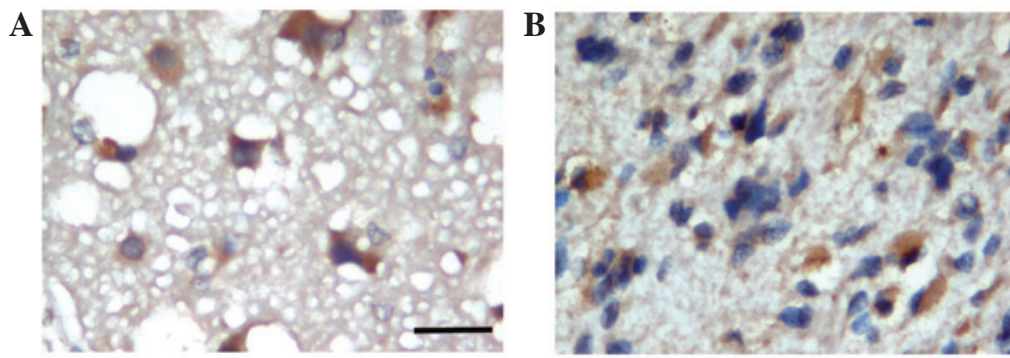

D

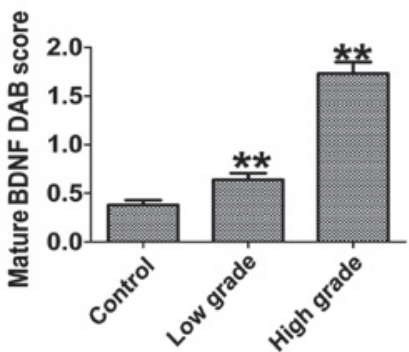

$\mathbf{E}$

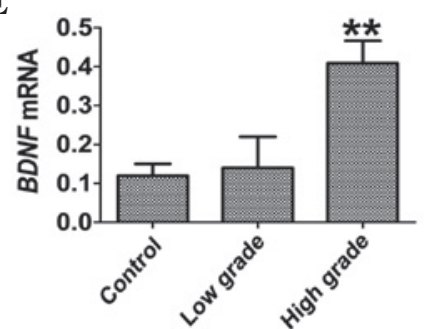

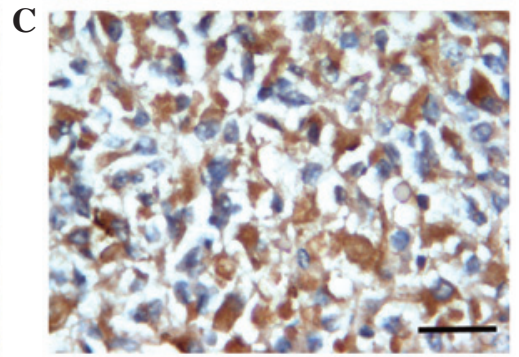

$\mathbf{F}$

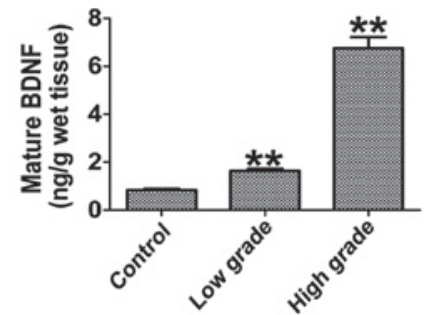

Figure 1. Expression of mature BDNF in human glioma tissues at various grades of malignancy. (A-C) IHC for mature BDNF in (A) control tissue, (B) low-grade glioma tissue and (C) high-grade glioma tissue (scale bar, $25 \mu \mathrm{m}$ ). (D) IHC scores for mature BDNF immunostaining. (E) Detection of BDNF mRNA by reverse transcription-quantitative polymerase chain reaction. (F) Findings of the ELISA assay. ${ }^{* *} \mathrm{P}<0.01$ vs. control. BDNF, brain-derived neurotrophic factor; IHC, immunohistochemistry; DAB, 3,3'-diaminobenzidine.

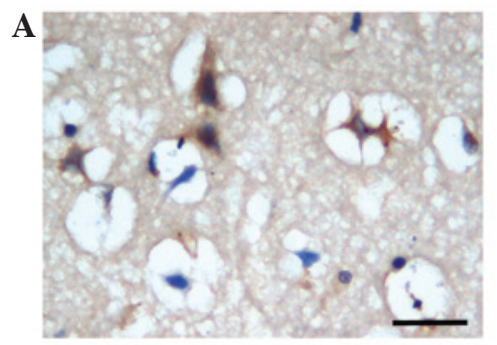

D

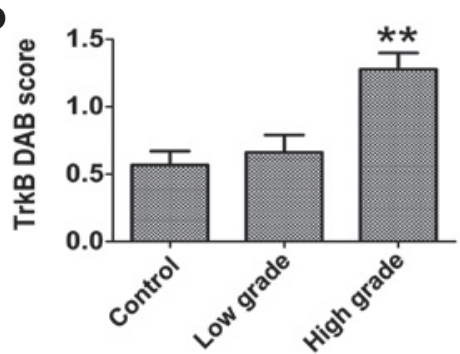

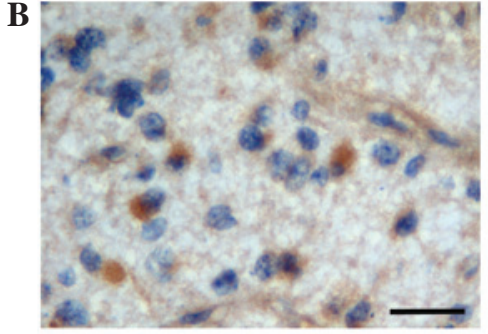

$\mathbf{E}$

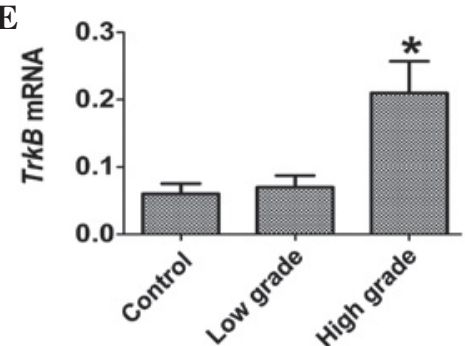

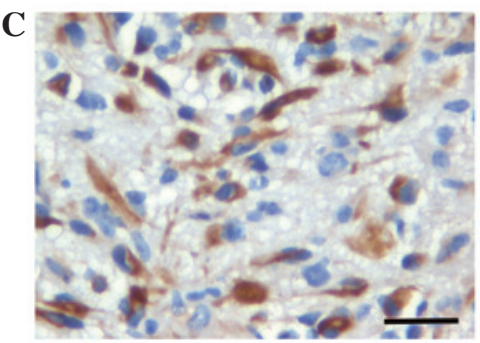

$\mathbf{F}$

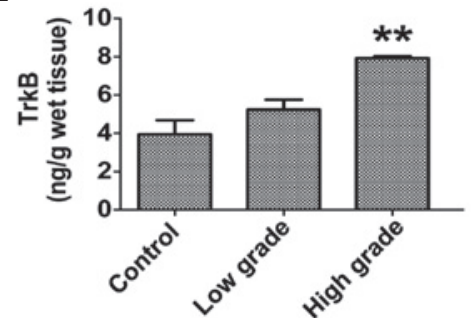

Figure 2. Expression of TrkB in human glioma tissues at various grades of malignancy. (A-D) IHC for TrkB in (A) control tissues, (B) low-grade glioma tissues and (C) high-grade glioma tissues (scale bar, $25 \mu \mathrm{m}$ ). (D) IHC scores for TrkB immunostaining. (E) Detection of TrkB mRNA by reverse transcription-quantitative polymerase chain reaction. (F) Findings of the ELISA assay. ${ }^{* *} \mathrm{P}<0.01,{ }^{*} \mathrm{P}<0.05$ vs. control. TrkB, tyrosine receptor kinase B; IHC, immunohistochemistry.

A

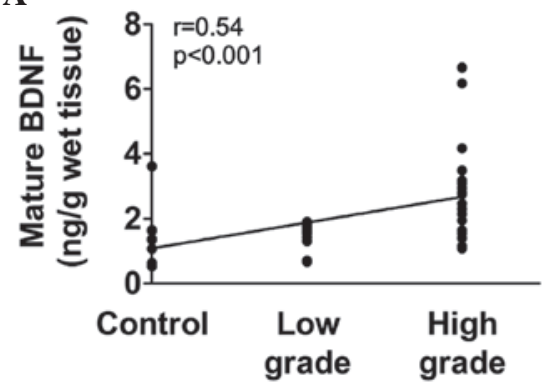

$\mathbf{B}$

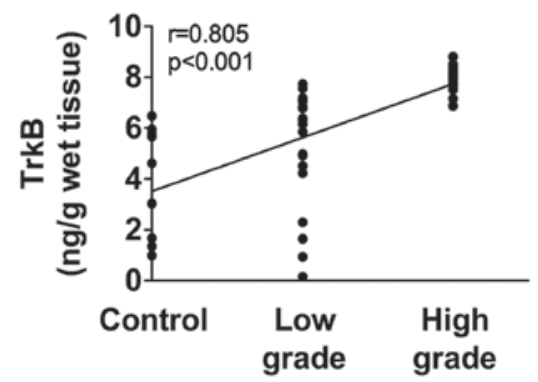

C

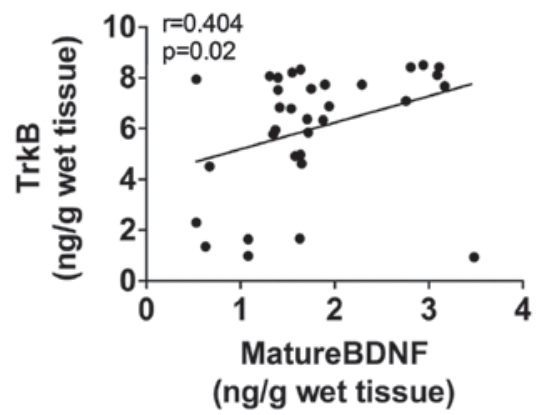

Figure 3. Correlation between mature BDNF and TrkB expression and glioma tumor grade. (A) Correlation between mature BDNF expression and the tumor grade. (B) Correlation between TrkB expression and tumor grade. (C) Correlation between the expression of mature BDNF and the expression of TrkB. BDNF, brain-derived neurotrophic factor; TrkB, tyrosine receptor kinase B. 
Correlation between the expression of mature BDNF and TrkB and the glioma malignancy grade. Spearman's rank correlation analysis revealed that mature BDNF $(r=0.54, \mathrm{P}<0.001)$ and TrkB $(r=0.805, P<0.001)$ were positively associated with the grade of malignancy in glioma (Fig. 3A and B). Spearman's rank correlation analysis also revealed that there was a positive correlation between the expression levels of mature $\mathrm{BDNF}$ and TrkB ( $r=0.404, \mathrm{P}=0.02$; Fig. 3C).

\section{Discussion}

The important role of the BDNF/TrkB signaling system in tumor cell proliferation and survival have been demonstrated in previous studies (1-3). The expression of BDNF is correlated with a poor prognosis in various TrkB-expressing human cancers, including tumors in the nervous system (1-3). Previous studies have revealed that BDNF mRNA is a prevalent transcript in 24 cell lines derived from human malignant gliomas (4).

ProBDNF and mature BDNF coexist in the CNS and perform opposite roles in neuronal functions through two transmembrane receptor signaling systems, consisting of p75NTR and TrkB (5-7). ProBDNF is a high-affinity functional ligand for the pro-apoptotic p75NTR, whereas the proteolytically cleaved mature BDNF is the preferred ligand for TrkB (6-7). A previous study has demonstrated that proBDNF negatively regulates the growth and migration of $\mathrm{C} 6$ glioma cells through p75NTR (16). It has also been demonstrated that mature BDNF induces C6 glioma cell proliferation and invasion in vitro (17). Considering the opposite effects on the biological behavior of gliomas cells in vitro, measurement of the level of mature BDNF alone in normal human brain tissue and glioma tissue samples in vivo may be informative.

The present study has revealed that mature BDNF and its receptor TrkB are highly expressed in human glioma cells using various approaches, consisting of IHC, ELISA and RT-qPCR assays. In the present study, the expression of TrkB was found to be upregulated in human high-grade gliomas, and the receptor is positively correlated with the grade of the gliomas. The present results are consistent with previous studies that reported the frequent robust expression of TrkB in highly invasive tumors (21) and the activity of TrkB as a key regulator of tumor malignancy. Notably, the expression profiles of mature BDNF and TrkB are similar, demonstrating increased expression parallel to the increase in gliomas grades. In addition, the co-localization of mature BDNF and TrkB in human gliomas was increased in high-grade samples, suggesting that the mature BDNF/TrkB signaling pathway plays a role in modulating the malignancy and prognosis of glioma. These results are consistent with the results of a previous study that reported the promotion of the proliferation, infiltration and migration of C6 glioma cells demonstrated by mature BDNF in vitro (17). The promotion of the proliferation, infiltration and migration of glioma cells by mature BDNF may be critical in the promotion of low-grade gliomas to over-aggressive high-grade gliomas. Suppression of the mature BDNF/TrkB signaling pathway by either specific mature BDNF neutralizing antibodies or TrkB-receptor antibodies may demonstrate therapeutic significance in high-grade glioma.
These results are consistent with previous findings that suggested the possibility of the proBDNF-p75-sortilin pathway being a balancing signal for tumor growth through the mature BDNF-TrkB pathway $(7,16)$. The balance between tumor cell death and survival may depend upon the proportions of mature and proBDNF available to cells expressing TrkB and p75NTR. ProBDNF-converting enzymes, such as MMPs or tissue plasminogen activator are produced and released by glioma cells $(22,23)$. In particular, these proteases have been reported to be closely associated with invasion and angiogenesis in malignant gliomas $(22,23)$. It would be of considerable interest to study the precise mechanisms controlling the relative expression levels of proBDNF and mature BDNF to limit or amplify distinct neurotrophin activities in the development of glioma.

Overall, the present data have indicated that increased levels of mature BDNF contribute notably to the development of malignancy in human gliomas through the primary BDNF receptor TrkB. The present study suggests that the suppression of the mature BDNF signaling pathway, but not the proBDNF signaling pathway, may be a novel therapeutic strategy for high-grade gliomas.

\section{Acknowledgements}

This study was supported by the National Key Basic Research Program of China (grant no., 2011CB944200).

\section{References}

1. Nakagawara A, Azar CG, Scavarda NJ and Brodeur GM: Expression and function of TRk-B and BDNF in human neuroblastomas. Mol Cell Biol 14: 759-767, 1994.

2. Stephan H, Zakrzewski JL, Bölöni R, Grasemann C, Lohmann DR and Eggert A: Neurotrophin receptor expression in human primary retinoblastomas and retinoblastoma cell lines. Pediatr Blood Cancer 50: 218-222, 2008.

3. Artico M, Bronzetti E, Pompili E, Ionta B, Alicino V, D'Ambrosio A, Santoro A, Pastore FS, Elenkov I and Fumagalli L: Immunohistochemical profile of neurotrophins in human cranial dura mater and meningiomas. Oncol Rep 21: 1373-1380, 2009.

4. Hamel W, Westphal M, Szönyi E, Escandón E and Nikolics K: Neurotrophin gene expression by cell lines derived from human gliomas. J Neurosci Res 34: 147-157, 1993.

5. Zhou XF, Song XY,Zhong JH, Barati S, Zhou FH and Johnson SM: Distribution and localization of pro-brain-derived neurotrophic factor-like immunoreactivity in the peripheral and central nervous system of the adult rat. J Neurochem 91: 704-715, 2004.

6. Seidah NG and Chretien M: Proprotein and prohormone convertases: A family of subtilases generating diverse bioactive polypeptides. Brain Res 848: 45-62, 1999.

7. Barker PA: Whither proBDNF?. Nat Neurosci 12: 105-106, 2009.

8. Teng HK, Teng KK, Lee R, Wright S, Tevar S, Almeida RD, Kermani P, Torkin R, Chen ZY, Lee FS, Kraemer RT, et al: ProBDNF induces neuronal apoptosis via activation of a receptor complex of p75NTR and sortilin. J Neurosci 25: 5455-5463, 2005.

9. Kenchappa RS, Zampieri N, Chao MV, et al: Ligand-dependent cleavage of the P75 neurotrophin receptor is necessary for NRIF nuclear translocation and apoptosis in sympathetic neurons. Neuron 50: 219-232, 2006.

10. Fan YJ, Wu LL, Li HY, Wang YJ and Zhou XF: Differential effects of pro-BDNF on sensory neurons after sciatic nerve transection in neonatal rats. Eur J Neurosci 27: 2380-2390, 2008.

11. Woo NH, Teng HK, Siao CJ, Chiaruttini C, Pang PT, Milner TA, Hempstead BL and Lu B: Activation of p75NTR by proBDNF facilitates hippocampal long-term depression. Nat Neurosci 8: 1069-1077, 2005.

12. Lu B: Pro-region of neurotrophins: Role in synaptic modulation. Neuron 39: 735-738, 2003.

13. Lu B, Pang PT and Woo NH: The yin and yang of neurotrophin action. Nat Rev Neurosci 6: 603-614, 2005. 
14. Koshimizu H, Hazama S, Hara T, et al: Distinct signaling pathways of precursor BDNF and mature BDNF in cultured cerebellar granule neurons. Neurosci Lett 473: 229-232, 2010.

15. Sun Y, Lim Y, Li F, Liu S, Lu JJ, Haberberger R, Zhong JH and Zhou XF: ProBDNF collapses neurite outgrowth of primary neurons by activating RhoA. PLoS One 7: e35883, 2012.

16. Xiong J, Zhou L, Yang M, Lim Y, Zhu YH, Fu DL, Li ZW, Zhong JH, Xiao ZC and Zhou XF: ProBDNF and its receptors are upregulated in glioma and inhibit the growth of glioma cells in vitro. Neuro Oncol 15: 990-1007, 2013.

17. Xiong J, Zhou L, Lim Y, Yang M, Zhu YH, Li ZW, Zhou FH, Xiao ZC and Zhou XF: Mature BDNF promotes the growth of glioma cells in vitro. Oncol Rep 30: 2719-2724, 2013.

18. Louis DN, Ohgaki H, Wiestler OD, Cavenee WK, Burger PC, Jouvet A, Scheithauer BW and Kleihues P: The 2007 WHO classification of tumours of the central nervous system. Acta Neuropathol 114: 97-109, 2007.
19. Livak KJ and Schmittgen TD: Analysis of relative gene expression data using real-time quantitative PCR and the 2 (-Delta Delta C (T)) Method. Methods 25: 402-408, 2001

20. Zhou L, Xiong J, Lim Y, Ruan Y, Huang C, Zhu Y, Zhong JH, Xiao Z and Zhou XF: Upregulation of blood proBDNF and its receptors in major depression. J Affect Disord 150: 776-784, 2013.

21. Assimakopoulou M, Kondyli M, Gatzounis G, Maraziotis T and Varakis J: Neurotrophin receptors expression and JNK pathway activation in human astrocytomas. BMC Cancer 7: 202, 2007.

22. Hagemann C, Anacker J, Ernestus RI and Vince GH: A complete compilation of matrix metalloproteinase expression in human malignant gliomas. World J Clin Oncol 3: 67-79, 2012.

23. Salmaggi A, Croci D, Prina P, Cajola L, Pollo B, Marras CE, Ciusani E, Silvani A, Boiardi A and Sciacca FL: Production and post-surgical modification of VEGF, tPA and PAI-1 in patients with glioma. Cancer Biol Ther 5: 204-209, 2006. 